\title{
Perfil da resiliência em indivíduos com amputação de membro inferior
}

\section{Profile of resilience in individuals with lower limb amputation}

\author{
(1) Maressa Gonçalves da Paz ${ }^{1}$, Juliana Caldas de Souza ${ }^{2}$, Fernanda Miranda de Oliveira ${ }^{3}$
}

\section{RESUMO}

Objetivo: Identificar o perfil sociodemográfico dos amputados de membro inferior bem como, os níveis e os fatores da resiliência. Método: Trata-se de um estudo descritivo, quantitativo e transversal. Os dados foram coletados através de um questionário semiestruturado após aprovação pelo Comitê de Ética e Pesquisa. O período de coleta foi entre Setembro a Novembro de 2017 no ambulatório do Centro Estadual de Reabilitação e Readaptação de Goiânia - Goiás. Os dados foram apresentados em frequências, média e desvio padrão. Resultados: Verificou-se que os indivíduos resilientes tinham amputação acima do joelho (44\%), de etiologia traumática (30,1\%), não receberam orientação após a amputação $(41,5 \%)$ e utilizam as muletas como principal dispositivo auxiliar na locomoção (49\%). Houve uma predominância da resiliência moderada 33 (62,2\%) e de respostas do tipo concordo para os fatores I e III da escala de resiliência. Para o fator II houve semelhança nas respostas do tipo nem concordo e nem discordo e do tipo concordo. Discussão: Amputação acima do nível do joelho, etiologia traumática e ausência de orientação quanto aos cuidados com o coto foram predominantes nos indivíduos resilientes tais características estão associadas a um maior grau de incapacidade, cuidados inadequados com o coto, stress e depressão. Acredita-se que condições adversas pode influenciar positivamente a resiliência graças a capacidade de ajustamento e adaptação do indivíduo. Conclusão: A resiliência moderada evidencia que os amputados possuem estratégias de enfretamento positivas, porém e necessário realizar programas de treinamento a fim de promover melhor independência e determinação.

Palavras-chave: Amputação, Extremidade Inferior, Reabilitação, Resiliência Psicológica

\footnotetext{
${ }^{1}$ Enfermeira Residente, Residência Multiprofissional em Saúde Funcional e Reabilitação - CRER.

${ }^{2}$ Enfermeira Tutora, Residência Multiprofissional em Saúde Funcional e Reabilitação - CRER.

${ }^{3}$ Enfermeira Preceptora, Residência

Multiprofissional em Saúde Funcional e Reabilitação - CRER.
}

\section{Correspondência}

Maressa Gonçalves da Paz

E-mail: mary-184@hotmail.com

Recebido em 21 Fevereiro 2018.

Aceito em 26 Outubro 2018.

\section{Como citar}

Paz MG, Souza JC, Oliveira FM. Perfil da resiliência em indivíduos com amputação de membro inferior. Acta Fisiatr. 2018;25(2):69-73.

\begin{abstract}
Objective: To identify the sociodemographic profile of lower limb amputees as well as levels and resilience factors. Method: This is a descriptive, quantitative and cross-sectional study. Data were collected through a semi-structured questionnaire after approval by the Research Ethics Committee. The collection period was from September to November 2017 at the outpatient clinic of the State Center for Rehabilitation and Readaptation of Goiânia - Goiás. Data were presented in frequencies, mean and standard deviation. Results: Resilient individuals were found to have above-knee amputation (44\%), traumatic etiology $(30.1 \%)$, received no orientation after amputation (41.5\%) and used crutches as the main auxiliary device in locomotion (49\%). There was a predominance of moderate resilience 33 (62.2\%) and "agree-type" responses to factors I and III of the resilience scale. For factor II there was similarity in the "neither agree- nor disagreetype", and "agree-type" answers. Discussion: Above-knee amputation, traumatic etiology, and lack of stump care guidance were prevalent in resilient individuals. These features are associated with a higher degree of disability, inadequate stump care, stress, and depression. It is believed that adverse conditions can positively influence resilience thanks to the individual's ability to adjust and adapt. Conclusion: Moderate resilience shows that amputees have positive coping strategies, but it is necessary to carry out training programs to promote better independence and determination.
\end{abstract}

Keywords: Amputation, Lower Extremity, Rehabilitation, Resilience, Psychological 


\section{INTRODUÇÃO}

Considerada um problema de saúde pública, ${ }^{1,2}$ a amputação afeta as condições físicas, emocionais, pessoais e sociais dos indivíduos, diminuindo assim a autonomia deles e tornando-os dependentes de outrem. ${ }^{3}$ Além disso, a amputação traz mudanças na capacidade funcional, prejudicando as atividades da vida diária e portanto a qualidade de vida. ${ }^{4,5}$

Em 2011 o Sistema Único de Saúde (SUS) registrou que de todas as amputações realizadas, $94 \%$ foram de membro inferior. ${ }^{6}$ $\mathrm{Na}$ Inglaterra a amputação de membro inferior foi registrada em 5.500 pessoas entre os anos de 2009 e 2010. Há ainda uma previsão de que em 2050 o índice de pessoas amputadas nos Estados Unidos, alcance 3,6 milhões de pessoas..$^{1,2}$

Relacionada a qualidade de vida, ${ }^{4,7}$ a resiliência é um constructo complexo e está sujeita à influencias culturais, genéticas e ambientais. ${ }^{8}$ Considerada um processo dinâmico, a resiliência investiga a capacidade de um indivíduo, família ou mesmo uma comunidade de responder de forma positiva/ saudável a uma ou a várias situações conflitantes. ${ }^{9,10}$ Níveis mais elevados de resiliência estão relacionados com maiores níveis de aceitação, independência e adaptação. ${ }^{11}$

Pesquisas acerca da resiliência na área da saúde tiveram seu início em 1970 com indivíduos em situações traumáticas agudas ou prolongadas. ${ }^{12}$ Atualmente diversos estudos visam compreender a resiliência em oncologia, ${ }^{13}$ nas alterações cardiovasculares, metabólicas, renais, ${ }^{14-16}$ na compreensão acerca do envelhecimento ${ }^{17}$ e na introdução da ideia de "gene resiliente". ${ }^{18,19}$ Porém, poucos são as pesquisas sobre a resiliência em amputados.

Incapacidades, deformidades, alterações psicossociais são algumas das consequências trazidas pela amputação. ${ }^{20}$ Superá-las com êxito poderá auxiliar no processo de reabilitação. Assim compreender a resiliência em indivíduos com amputação bem como a identificação de características relacionadas a resiliência poderá auxiliar na compreensão dos mecanismos de enfrentamento utilizado pelos amputados.

\section{OBJETIVO}

Identificar o perfil de indivíduos com amputação de membro inferior, os níveis de resiliência (baixo, moderado e alto) e seus fatores.

\section{MÉTODOS}

Trata-se de um estudo descritivo, quantitativo e transversal. A amostra foi por conveniência, sendo obtida no ambulatório de amputados do Centro Estadual de Reabilitação e Readaptação Doutor Henrique Santillo de Goiânia, Goiás. O estudo foi aprovado no Comitê de Ética Pesquisa (CEP) com o seguinte número de protocolo $\mathrm{n}$ - 2.203.642.

A coleta foi realizada no primeiro atendimento do amputado ao centro especializado. O período foi compreendido de setembro a novembro de 2017. Após a assinatura do Termo de Consentimento Livre Esclarecido (TCLE), os dados sociodemográficos, clínicos e a escala de resiliência foram obtidos via instrumento semiestruturado.

A escala de resiliência foi desenvolvida por Wagnild \& Young. ${ }^{21}$ No Brasil, esta escala foi validada e adaptada em 2005 por Pesce et al. ${ }^{22} \mathrm{~A}$ escala de resiliência é composta por 25 itens (perguntas) com resposta tipo Likert. A pontuação mínima na escala é de 25 e a máxima de 175 pontos. As perguntas da escala podem ser divididas em três grupos distintos entre si pelo fator de resiliência. Fator I - Resolução de Ações e Valores; II - Independência e Determinação; III - Autoconfiança e Capacidade de Adaptação à Situação. Para a classificação da resiliência serão considerados: baixa resiliência (escores menores que 125), resiliência moderada (escores entre 125 e 145) e resiliência alta (escore acima de 145). ${ }^{22}$

Os dados foram tabulados na planilha do Excel $^{\circ}$ for Windows e a distribuição de frequência absoluta e relativa, medidas de tendência central (média) e o desvio padrão foram analisados pelo Statistical Package for the Social Sciences (SPSS) versão 24.

Para análise de cada fator da escala de resiliência foi a utilizada a média de respostas de cada indivíduo às perguntas correspondentes ao fator. Após a realização dessa média, os indivíduos foram classificados de acordo com a pontuação na escala Likert. Foi considerado indivíduos que discordam os que tiveram média entre 1 e 3,9; os que nem concordam e nem discordam aqueles cuja média está entre 4 e 4,9; e os que concordam os que possuem média entre 5 e 7 .

\section{RESULTADOS}

Os dados sociodemográficos são apresentados na Tabela 1. A amostra desse estudo foi de 53 indivíduos com idade média de 51,4 anos. Houve uma predominância de $66 \%$ indivíduos do sexo masculino, $35,8 \%$ solteiros, $37,7 \%$ caucasianos, $41,6 \%$ com ensino fundamental incompleto, $41,6 \%$ com Diabetes Mellitus seguindo de $36,6 \%$ com Hipertensão Arterial Sistêmica e 73,6\% pertencentes a classe social E. O Instituto Brasileiro de Geografia e Estatística (IBGE) define como classe social $E$ aquela que recebe até dois salários mínimos. ${ }^{23}$

As características acerca da amputação dos indivíduos resilientes e com baixa resiliência foram apresentadas na Tabela 2 . Os indivíduos com resiliência moderada e alta foram considerados resilientes. Houve prevalência dos indivíduos resilientes com amputação acima do nível do joelho (44\%) e de etiologia traumática $(30,1 \%)$. O intervalo de tempo após a amputação e a primeira consulta em uma unidade de reabilitação foi superior a um ano (média de 20 meses). Dos indivíduos resilientes $41,5 \%$ não receberam orientação quanto aos cuidados com o coto após a amputação e $69,8 \%$ utilizaram as muletas e o andador como principal método de auxílio a locomoção.

Quanto aos níveis de resiliência houve maior predominância de indivíduos com resiliência moderada (62,2\%). Já em relação aos fatores da resiliência houve predominância de indivíduos que tiveram respostas do tipo concordam (Tabela 3).

\section{DISCUSSÃO}

Indivíduos do sexo masculino, caucasianos, com média de idade de 49,4 a 53,1 anos, com renda abaixo de 4 salários mínimos, ensino fundamental incompleto e solteiros são semelhanças nos estudos com amputados. ${ }^{24,25}$

Amputação acima do nível do joelho, etiologia traumática e a ausência de orientação quanto aos cuidados com o coto são três características que foram predominantes nos indivíduos resilientes. Resultados semelhantes foram encontrados em outros estudos. ${ }^{26-28}$ Entretanto, tais características estão associadas a um maior grau de incapacidade, cuidados inadequados com o coto, stress e depressão. ${ }^{29,30}$

Estudos tem evidenciado que a presença das condições adversas pode influenciar positivamente a resiliência. Indivíduos com maior vulnerabilidade e alta exposição ao stress apresentam maior resiliência, graças a capacidade de ajustamento e adaptação perante a uma situação de vulnerabilidade. ${ }^{31-34}$

O Diabetes Mellitus e a Hipertensão Arterial Sistêmica foram significativas nos amputados e cerca de $11,6 \%$ possuíam mais de uma comorbidade. Esses dados foram 
Tabela 1. Dados sociodemográficos e principais comorbidades

\begin{tabular}{|c|c|}
\hline Características & $\mathrm{N}=53$ \\
\hline \multicolumn{2}{|l|}{ Sexo } \\
\hline Masculino & $35(66 \%)$ \\
\hline Feminino & $18(34 \%)$ \\
\hline \multicolumn{2}{|l|}{ Estado Civil } \\
\hline Solteiro & $19(35,9 \%)$ \\
\hline Casado & $18(34 \%)$ \\
\hline Divorciado & $10(18,9 \%)$ \\
\hline Viúvo & $6(11,3 \%)$ \\
\hline \multicolumn{2}{|l|}{ Idade } \\
\hline Adolescência (15 - 21) & $1(1,9 \%)$ \\
\hline Adulto (22- 64) & $37(69,8 \%)$ \\
\hline Idoso $\geq 65$ anos & $15(28,3 \%)$ \\
\hline Média (DP); mín-máx & $51,4(16,7) ; 20-80$ \\
\hline \multicolumn{2}{|l|}{ Ełnia } \\
\hline Branco & $20(37,7 \%)$ \\
\hline Pardos & $16(30,2 \%)$ \\
\hline Negro & $13(24,5 \%)$ \\
\hline Amarelo & $4(7,5 \%)$ \\
\hline \multicolumn{2}{|l|}{ Escolaridade } \\
\hline Fundamental Incompleto & $27(50,9 \%)$ \\
\hline Fundamental Completo & $6(11,3 \%)$ \\
\hline Médio incompleto & $6(11,3 \%)$ \\
\hline Médio completo & $9(17 \%)$ \\
\hline Superior incompleto & $2(3,8 \%)$ \\
\hline Superior completo & $3(5,7 \%)$ \\
\hline \multicolumn{2}{|l|}{ Classe Social } \\
\hline Sem renda & $8(15,1 \%)$ \\
\hline Classe E & $39(73,6 \%)$ \\
\hline Classe D & $6(11,3 \%)$ \\
\hline Comorbidades & $N=60$ \\
\hline Diabetes Mellitus & $25(41,6 \%)$ \\
\hline Hipertensão Arterial Sistêmica & $22(36,6 \%)$ \\
\hline Outras* & $13(21,6 \%)$ \\
\hline
\end{tabular}

*Outras comorbidades: insuficiência renal, hipo ou hipertiroidismo, arritmias, osteoporose e outras

Tabela 2. Características clínica da amputação em indivíduos resilientes e não resilientes

\begin{tabular}{lcc}
\hline Características & Baixa Resiliência & Resilientes \\
\cline { 2 - 3 } Nível de amputação & & $\mathbf{N}=\mathbf{5 3}$ \\
Nível acima do joelho & $8(13,5 \%)$ & $26(44 \%)$ \\
Nível abaixo do joelho & $9(15,2 \%)$ & $16(27,2 \%)$ \\
Causas da amputação & & $7(13,2 \%)$ \\
Alteração vascular & $4(7,5 \%)$ & $10(18,9 \%)$ \\
Diabetes & $3(5,7 \%)$ & $16(30,1 \%)$ \\
Trauma & $5(9,4 \%)$ & $2(3,8 \%)$ \\
Câncer & - & $1(1,9 \%)$ \\
Infeção & - & $3(5,7 \%)$ \\
Outra* & $2(3,8 \%)$ & $17(32,1 \%)$ \\
Orientação após a amputação** & & $22(41,5 \%)$ \\
Sim & $4(7,5 \%)$ & \\
Não & $10(18,9 \%)$ & $37(69,8 \%)$ \\
Dispositivo auxiliar para locomoção & & $2(3,8 \%)$ \\
Muleta axilar, canadense e andador & $11(20,7 \%)$ & \\
Cadeira de rodas & $3(5,6 \%)$ & \\
Tempo em meses de atendimento & $20(28,4) ; 8 ; 2-144$ & \\
Média (DP); mediana; mín-máx & & \\
\hline
\end{tabular}

*Outras etiologias: má formação congênita e iatrogênicas; ${ }^{* *}$ Orientação para os cuidados com o coto após amputação: dessensibilização e posicionamento do coto, fortalecimento de segmentos corporais, controle de edema semelhantes aos encontrados nos estudos de Silva, ${ }^{35}$ Borges $^{36}$ e podem indicar um risco para novas amputações. Segundo a Carga Global de Doença (GBD - Global Burden of Diseases) essas comorbidades estão associadas a um aumento de incapacidades e consequentemente de anos de vida sadia perdidos. ${ }^{37}$

Dos indivíduos resilientes, $69,8 \%$ utilizavam como principal dispositivo auxiliar na locomoção a muleta axilar ou muleta canadense ou andador. Estudos evidenciam que as muletas trazem maior autonomia e independência para os indivíduos. ${ }^{38}$ Os benefícios trazidos pelas tecnologias podem ser considerados como um fator de proteção para a resiliência. Os fatores de proteção são recursos que minimizam os efeitos adversos decorrentes das situações traumáticas ou conflitantes. ${ }^{39,40}$

O período decorrido entre a amputação e o primeiro atendimento em uma unidade de reabilitação foi de um ano e nove meses. A literatura tem evidenciado uma média de tempo prolongada. ${ }^{41}$ Essa espera por atendimento em uma unidade especializada mostra uma fragilidade na rede de atenção à pessoa com amputação, além de apontar para uma possível burocratização no encaminhamento aos centros e/ou unidades de reabilitação. ${ }^{42}$

Quanto ao nível de resiliência, 62,2\% tiveram a resiliência moderada. Resultados semelhantes foram encontrados por Mendoza \& Espinoza ${ }^{43}$ e Cardoso \& Sacomori. ${ }^{44}$ Estudos que utilizam a mesma escala e a mesma população são poucos, o que dificulta outras comparações. Acreditase que o nível de amputação acima do joelho, falta de orientação quanto aos cuidados com o coto e a etiologia traumática tenham proporcionado maior exposição as condições de vulnerabilidade e consequentemente influenciado na resiliência desses indivíduos. O que significa que apesar das características insatisfatórias associada a amputação, os indivíduos resilientes estão superando de forma positiva e saudável situações de adversidade ligadas a perca do membro. ${ }^{45}$

Quantos aos fatores de resiliência, no fator II (Independência e Determinação) houve semelhança no percentual de respostas do tipo nem concordo e nem discordo e do tipo concordo. No fator I (Resolução de Ações e Valores) e III (Autoconfiança e Capacidade de Adaptação à Situação) houve predominância das repostas do tipo concordo. Isso mostra que os amputados apresentam potencialidades nas resoluções de ações e valores e na autoconfiança e capacidade de adaptação à situação, dando 
Tabela 3. Valores absolutos e relativos dos níveis de resiliência e das respostas por domínios

\begin{tabular}{|c|c|c|c|}
\hline Níveis de Resiliência & \multicolumn{3}{|c|}{$\mathrm{N}=53(100 \%)$} \\
\hline Resiliência Baixa & \multicolumn{3}{|c|}{$14(26,4 \%)$} \\
\hline Resiliência Moderada & \multicolumn{3}{|c|}{$33(62,2 \%)$} \\
\hline Resiliência Alta & \multicolumn{3}{|c|}{$6(11,3 \%)$} \\
\hline & \multicolumn{3}{|c|}{ Indivíduos por categoria de resposta na escala Likert } \\
\hline Fator de Resiliência & $\begin{array}{c}\text { (a) Discordo } 1^{*}, \\
2^{*}, 3^{*}\end{array}$ & (b) Nem concordo nem discordo $4^{*}$ & (c) Concordo $5^{*}, 6^{*}, 7^{*}$ \\
\hline Fator I: Resolução de Ações e Valores & $1(1,8 \%)$ & $8(15 \%)$ & 44 (83\%) \\
\hline $\begin{array}{l}\text { Fator III Independência e } \\
\text { Determinação }\end{array}$ & $14(26,4 \%)$ & $19(35,8 \%)$ & $20(37,7 \%)$ \\
\hline $\begin{array}{l}\text { Fator III: Autoconfiança e Capacidade } \\
\text { de Adaptação à Situação }\end{array}$ & $3(5,6 \%)$ & $12(22,6 \%)$ & 38 \\
\hline
\end{tabular}

(a) indivíduos com média entre 1 e 3,9 nas respostas. (b) indivíduos com média entre 3 e 4,9 nas respostas. (c) indivíduos com média entre 5 e 7 nas respostas. 1*- Discordo Totalmente; 2*- Discordo muito; $3^{*}$ - Discordo pouco; $4 *$ - Nem concordo nem discordo; $5 *$ - Concordo pouco; 6*- Concordo muito; $7^{*}$ - Concordo totalmente

significado para as adversidades e lidando de maneira positiva com os conflitos e adversidades. O percentual significativo de respostas nem concordo e nem discordo no fator independência e determinação indica a necessidade de estímulo dessa competência a partir de programas de treinamento com equipe multiprofissional especializada.

\section{CONCLUSÃO}

Os dados sociodemográficos são similares aos encontrados na literatura. Os amputados possuem uma resiliência moderada e predominância de respostas do tipo concordo para os fatores I (Resolução de Ações e Valores) e III (Autoconfiança e Capacidade de Adaptação à Situação). Para o fator II houve semelhança de respostas do tipo nem concordo e nem discordo com respostas do tipo concordo.

A resiliência é um constructo complexo que pode ser desenvolvido e está sujeito a variações ambientais, pessoais e culturais. É uma ferramenta que pode auxiliar no enfretamento das incapacidades e consequências trazidas pela perca de um membro. O perfil da resiliência moderada nos amputados de membro inferior evidencia que esses indivíduos desenvolveram recurso a fim de superar a perca de um membro, porém é necessário monitorar esses indivíduos a fim de garantir o bem - estar e o equilíbrio psicológico e realizar programas de treinamento com a finalidade de aprimorar o nível da resiliência.

Estudos acerca da resiliência em amputados são escassos na literatura nacional. Esperamos com esse trabalho contribuir de forma significativa com o processo de reabilitação dos amputados e sugerimos ainda que novos estudos sejam realizados acerca dessa temática.

\section{REFERÊNCIAS}

1. Varma P, Stineman MG, Dillingham TR. Epidemiology of limb loss. Phys Med Rehabil Clin N Am. 2014;25(1):1-8. DOI: https://doi.org/10.1016/j. pmr.2013.09.001

2. Ziegler-Graham K, MacKenzie EJ, Ephraim PL, Travison TG, Brookmeyer R. Estimating the prevalence of limb loss in the United States: 2005 to 2050. Arch Phys Med Rehabil. 2008;89(3):422-9. DOI: https://doi. org/10.1016/j.apmr.2007.11.005

3. Ali S, Fatima Haider SK. Psychological adjustment to amputation: variations on the bases of sex, age and cause of limb loss. J Ayub Med Coll Abbottabad. 2017;29(2):303-7.

4. Chamlian TR, Starling M. Avaliação da qualidade de vida e função em amputados bilaterais de membros inferiores: revisão da literatura. Acta Fisiátr. 2013;20(4): 229-33.

5. Knežević A, Salamon T, Milankov $M$, Ninković $S$, Jeremić Knežević $M$, Tomašević Todorović $S$ Assessment of quality of life in patients after lower limb amputation. Med Pregl. 2015;68(3-4):103-8. DOI: https://doi.org/10.2298/MPNS1504103K

6. Brasil. Diretrizes de atenção à pessoa amputada. Brasília: Ministério da Saúde; 2013.

7. Xu J, Ou L. Resilience and quality of life among Wenchuan earthquake survivors: the mediating role of social support. Public Health. 2014;128(5):430-7. DOI: https://doi.org/10.1016/j.puhe.2014.03.002

8. Rutter M. Implications of resilience concepts for scientific understanding. Ann N Y Acad Sci. 2006;1094:1-12. DOI: https://doi.org/10.1196/ annals.1376.002

9. World Health Organization. Regional Office for Europe. Building resilience: a key pillar of health 2020 and the sustainable development goals: examples from the WHO small countries initiative. Copenhagen: WHO; 2017.

10. Sociedade Brasileira de Resiliência. Guia rápido: o que é resiliência [texto da internet]. São Paulo: SOBRARE [citado 2018 Jan 31]. Disponível em: http://sobrare. com.br/

11. Wu G, Feder A, Cohen H, Kim JJ, Calderon S, Charney DS, et al. Understanding resilience. Front Behav Neurosci. 2013;7:10. DOI: https://doi.org/10.3389/ fnbeh.2013.00010

12. Brandão JM, Mahfoud M, Gianordoli IF. A construção do conceito de resiliência em psicologia: discutindo as origens. Paidéia. 2011;21(49): 263-71.
13. Tomaz LA, Veras Junior EF, Carvalho PMG Enfrentamento e resiliência de pacientes com câncer submetidos a tratamento quimioterápico. Rev Interd. 2015;8(2):195-201.

14. Lemos CMM, Moraes DW, Pellanda LC. Resilience in patients with ischemic heart disease. Arq Bras Cardiol. 2016;106(2):130-5. DOI: https://doi.org/10.5935/ abc. 20160012

15. Tavares BC, Barreto FA, Lodetti ML, Silva DMGV, Lessmann JC. Resiliência de pessoas com Diabetes Mellitus. Texto Contexto Enferm. 2011;20(4):751-7. DOI: https://doi.org/10.1590/\$0104-07072011000400014

16. Santos RI, Costa ORS. Avaliação da Resiliência em Pacientes com Insuficiência Renal Crônica Submetidos à Hemodiálise. Rev Ciên Saúde.2016;6(1):1-7.

17. Fontes AP, Neri AL. Resiliência e velhice: revisão de literatura. Ciência \& Saúde Coletiva. 2015;20(5):1475-95.

18. Feder A, Nestler EJ, Charney DS. Psychobiology and molecular genetics of resilience. Nat Rev Neurosci. 2009;10(6):446-57. DOI: https://doi.org/10.1038/nrn2649

19. Gillespie CF, Phifer J, Bradley B, Ressler KJ. Risk and resilience: genetic and environmental influences on development of the stress response. Depress Anxiety. 2009;26(11):984-92. DOI: https://doi. org/10.1002/da.20605

20. Sahu A, Sagar R, Sarkar S, Sagar S. Psychological effects of amputation: A review of studies from India. Ind Psychiatry J. 2016;25(1):4-10. DOI: https://doi. org/10.4103/0972-6748.196041

21. Wagnild GM, Young HM. Development and psychometric evaluation of the Resilience Scale. J Nurs Meas. 1993;1(2):165-78.

22. Pesce RP, Assis SG, Avanci JQ, Santos NC, Malaquias JV, Carvalhaes R. Adaptação transcultural, confiabilidade e validade da escala de resiliência. Cad Saude Publica. 2005;21(2):436-48. DOI: https://doi. org/10.1590/S0102-311X2005000200010

23. Instituto Brasileiro de Geografia e Estatística. Renda domiciliar per capita de 2016 [texto da internet]. Brasília: IBGE [citado 2017 Dez 12]. Disponível em: https://ww2.ibge.gov.br/home/estatistica/ indicadores/trabalhoerendimento/pnad_continua/ default_renda_percapita.shtm

24. Cassefo V, Nacaratto DC, Chamlian TR. Perfil epidemiológico dos pacientes amputados do Lar Escola São Francisco - estudo comparativo de 3 períodos diferentes. Acta Fisiátr.2003;10(2):67-71.

25. Lima LB, Correia VD, Salimene ACM. Perfil social do paciente amputado em processo de reabilitação. Acta Fisiátr. 2016;23(2):57-60.

26. Santos LF, Fritzen PG, Gonçalves BR, Melo SA, Silva VF. Perfil das amputações de membros inferiores de pacientes cadastrados na Associação de deficientes físicos de Apucarana. Rev Saúde Pesq. 2010;3(1):59-64.

27. Ramirez C, Menaker J. Traumatic amputations. Trauma Reports.2017;18(3):40-8.

28. Jadeja B, Zafer A, Jindal R, Gaur K. Profile of Lower Limb Amputees attended at a tertiary care Hospital: A Descriptive study. Int Multispecialty J Health. 2016;1(1);18-25.

29. MacKenzie EJ, Bosse MJ, Castillo RC, Smith DG, Webb LX, Kellam JF, et al. Functional outcomes following trauma-related lower-extremity amputation. J Bone Joint Surg Am. 2004;86-A(8):1636-45. DOI: https:// doi.org/10.2106/00004623-200408000-00006

30. Perkins ZB, De'Ath HD, Sharp G, Tai NR. Factors affecting outcome after traumatic limb amputation. Br J Surg. 2012;99 Suppl 1:75-86. DOI: https://doi. org/10.1002/bjs.7766

31. Werner EE. Resilience in development. Curr Dir Psychol Sci. 1995;4(3):81-5. DOI: https://doi.org/10.1111/14678721.ep10772327 
32. Unwin J, Kacperek L, Clarke C. A prospective study of positive adjustment to lower limb amputation. Clin Rehabil. 2009;23(11):1044-50. DOI: https://doi. org/10.1177/0269215509339001

33. Garmezy N. Resiliency and vulnerability to adverse developmental outcomes associated with poverty. Am Behav Sci. 1991;34(4):416-30. DOI: https://doi. org/10.1177/0002764291034004003

34. Xia ZY, KongY, Yin TT, Shi SH, Huang Rong, Cheng $\mathrm{YH}$. The impact of acceptance of disability and psychological resilience on post-traumatic stress disorders in burn patients. Int J Nurs Sci.2014;1(4):371-5. DOI: https://doi.org/10.1016/j. ijnss.2014.10.018

35. Jesus-Silva SG, Oliveira JP, Brianezi MHC, Silva MAM, Krupa AE, Cardoso RS. Análise dos fatores de risco relacionados às amputações maiores e menores de membros inferiores em hospital terciário. J Vasc Bras. 2017;16(1):16-22. DOI: https://doi. org/10.1590/1677-5449.008916
36. Borges WR. Fatores de risco para amputação em pacientes com isquemia crítica crônica dos membros inferiores. J Vasc Bras. 2017;16(2):180-1.

37. World Health Organization. The global burden of disease [text on the Internet]. Geneva: WHO [cited 2018 Jun 24]. Available from: http://www.who.int/ gho/mortality_burden_disease/en/

38. Stokes D, Curzio J, Berry A, Bacon E, Morten M Barker L. Pre prosthetic mobility: the amputees' perspectives. Disabil Rehabil. 2009;31(2):138-43. DOI: https://doi.org/10.1080/09638280701795543

39. Vetrayan J, Ghafar NBA, Paulra SJPV, Murad MS Occupational performance role and satisfaction among lower limb amputees with different adaptive devices usage. Procedia - Soc Beh Sci.2016;222(1):432-41. DOI: https://doi.org/10.1016/j.sbspro.2016.05.205

40. Murray c. Risk factors, protective factors, vulnerability, and resilience: A framework for understanding and supporting the adult transitions of youth with high
41. Schoeller AR, Silva DMGV, Vargas MAO, Borges AMF, Pires DEP, Bonetti A. Características das pessoas amputadas atendidas em um centro de reabilitação. Rev Enferm UFPE. 2013;7(2):445-51.

42. Vargas MAO, Ferrazzo S, Schoeller SD, Drago LC, Ramos FRS. Rede de atenção à saúde à pessoa amputada. Acta Paul Enferm. 2014;27(6):526-32. DOI: https://doi.org/10.1590/1982-0194201400086

43. Vargas Mendoza JE, Villavicencio Espinoza JM. Niveles de resiliencia en pacientes recientemente amputados: Consejos para fortalecerla. Centr Regi Inves Psicol 2011;5(1):13-20.

44. Cardoso FL, Sacomori C. Resilience of athletes with physical disabilities: a cross-sectional study. Rev Psicol Deporte. 2014;23(1):15-22.

45. Tugade MM, Fredrickson BL. Resilient individuals use positive emotions to bounce back from negative emotional experiences. J Pers Soc Psychol. 2004;86(2):320-33. DOI: https://doi.org/10.1037/00223514.86 .2 .320 